Conclusion* This case posed diagnostic and management dilemmas whether it is purely a cancer of the cervix or still representing an intestinal primary. The cancer is not HPV related so it can slip through the net of cervical screening and the main distinguished clinical feature was profuse mucus vaginal discahrge which greatly reduced after radiotherapy. The combination of neoadjuvant chemotherapy follwed by surgery was successful in this case. These enteric type cervical cancer represent a rare heterogenous group of cancers with variable presenations and prognosis.

\section{DOWNREGULATION OF LIMA1EXPRESSION INDUCES CANCER FEATURES IN NORMAL CERVICAL KERATINOCYTE HCK1T CELLS LEADING TO INCREASED MIGRATION AND INVASION}

${ }^{1}$ E Drakopoulou, ${ }^{1} E$ Kalafati*, ${ }^{1} \mathrm{AG}$ Vasilopoulou, ${ }^{1} \mathrm{~N}$ Anagnou, ${ }^{1 ; 2 \mathrm{~K}}$ Pappa. ${ }^{1}$ Biomedical Research Foundation of the Academy of Athens (BRFAA), Athens, Greece; ${ }^{2}$ University of Athens, First Department of Obstetrics and Gynecology, Athens, Greece

\subsection{6/ijgc-2021-ESGO.94}

Introduction/Background* Cervical cancer (CC) remains the fourth most common and lethal cancer type in women; therefore, a comprehensive understanding of the underlying mechanisms and development of novel diagnostic, prognostic and therapeutic approaches are critical steps for improving CC management. We have previously performed a detailed comparative proteomic analysis between $\mathrm{HeLa}, \mathrm{SiHa}$ and CD33A CC cell lines and the normal cervical keratinocytes HCK1T cells (Pappa et al, Oncol Rep. 42:1441, 2019) aiming to elucidate novel and potential therapeutic targets. Among hundreds of deregulated proteins between cancer and normal cell lines, LIMA1 or EPLIN, a protein decreased or lost in neoplasia, was statistically downregulated in all CC cell lines. Since LIMA1 role in CC is not fully elucidated yet, we aimed to downregulate its expression in HCK1T cells and assess the putative induction of cancer characteristics, such as increased proliferation, migration and invasion. Additionally, we studied the global m6A RNA methylation pattern in the presence and absence of LIMA1, aiming to highlight a potential role of epitranscriptomics in cancer onset and progression.

Methodology We downregulated LIMA1 expression in the HCK1T cell line using a lentiviral vector (LV) carrying a shRNA for either LIMA1 (shLIMA1) or no target (shNT) at a multiplicity of infection (MOI) 25. Following LIMA1 quantification both at protein and mRNA level, we assessed proliferation, invasion, migration and colony formation efficiency in shNT or shLIMA1 LV-transduced HCK1T cells. Proliferation was assessed using CCK8, while invasion and migration using Transwell $^{\circledR}$, with or without matrigel-coated inserts, respectively. Qualitative and quantitative assessment of m6A RNA methylation was done by $\mathrm{qPCR}$ and colorimetric EpiQuick ${ }^{\circledR}$ kit respectively.

Result(s)* Transduction with shLIMA1 LV led to a 98\% mean decrease of LIMA1 expression $(n=3)$, leading to a $126 \%$ $(\mathrm{p}=0.021, \mathrm{n}=3)$ and $103 \% \quad(\mathrm{p}=0.0091, \mathrm{n}=3)$ increase in migration and invasion respectively, while we did not observe any difference in cell proliferation and colony formation efficiency. Preliminary data regarding m6A RNA methylation point towards a slight increase following LIMA1 knockdown. Conclusion* LIMA1 may play an important role in cancer progression and metastasis and can set the grounds for novel and personalised therapy treatments for CC patients.

\section{6 THE DIFFERENTIAL EXPRESSION OF LIMA1/EPLIN IN NORMAL AND CERVICAL CANCER TISSUES}

${ }^{1}$ E Kalafati ${ }^{*},{ }^{1}$ E Drakopoulou, ${ }^{1} \mathrm{~N}$ Anagnou, ${ }^{1 ; 2}{ }^{2} \mathrm{~K}$ Pappa. ${ }^{1}$ Biomedical Research Foundation of the Academy of Athens (BRFAA), Athens, Greece; ${ }^{2}$ First Department of Obstetrics and Gynecology of the University of Athens, Athens, Greece

\subsection{6/ijgc-2021-ESGO.95}

Introduction/Background* LIMA1 or EPLIN is a cytoskeletonassociated protein involved in cytoskeleton regulation and dynamics, by inhibiting actin filament depolymerization and cross-linking filaments. The role of LIMA1 has been investigated in several malignant diseases including prostate, esophageal, breast and ovarian cancer. Its expression is often lost or aberrant in tumor cells leading to aggressive phenotypes. The aim of this study was to systematically assess the expression of LIMA1 in cervical cancer.

Methodology Immunohistochemical analysis using anti-EPLIN antibody was performed in 22 cases of cervical intraepithelial neoplasia, 14 cases of adenocarcinoma including one case with mucinous and one case with endometrioid adenocarcinoma, 5 cases of adenosquamous carcinoma, 25 cases of squamous cell carcinoma and 11 normal samples. The correlation of LIMA1 aberrant expression and disease progression was performed using the medical records of patients. Clinical samples of normal and tumor tissues were surgically excised, following an informed consent from all participants.

Result(s)* The levels of LIMA1 expression were low for healthy specimens, and limited to the cells of the basal membrane. LIMA1 expression showed a slight increase in the cases of intraepithelial neoplasia; however, it was quite low in cells displaying a HPV cytopathic effect (koilocytosis). On the contrary, the LIMA1 levels of both cytoplasmic and cellmembrane staining were significantly increased in the cases of adenocarcinoma and squamous cell carcinoma. Lastly, LIMA1 was highly expressed in undifferentiated, non-keratinizing, squamous cell carcinoma associated with poor prognosis.

Conclusion* Our results demonstrate that LIMA1 is differentially expressed not only in normal and cervical cancer tissues, but also in precancerous lesions and malignant cervical neoplasia, highlighting its role in cervical cancer progression and its potential exploitation as a prognostic factor.

\section{ABERRANT EXPRESSION OF ENZYMES REGULATING M6A RNA METHYLATION IN CERVICAL CANCER CELL LINES, AND THEIR IMPLICATION TO CERVICAL CANCER}

${ }^{1}$ E Drakopoulou, ${ }^{1} E$ Kalafati ${ }^{*},{ }^{1} \mathrm{AG}$ Vasilopoulou, ${ }^{1} \mathrm{~N}$ Anagnou, ${ }^{1 ; 2} \mathrm{~K}$ Pappa. ${ }^{1}$ Biomedical Research Foundation of the Academy of Athens (BRFAA), Athens, Greece; ${ }^{2}$ First Department of Obstetrics and Gynecology of the University of Athens, Athens, Greece

\subsection{6/ijgc-2021-ESG0.96}

Introduction/Background* RNA modifications play a key role in the regulation of gene expression. Among them, N6methyladenosine (m6A) is the most prevalent mRNA modification. m6A methylation modulates the gene expression by influencing numerous aspects of mRNA metabolism, including pre-mRNA processing, nuclear export, and translation. Many studies have demonstrated the importance of m6Amediated post-transcriptional gene regulation in numerous physiological and pathophysiological processes, especially in 